\title{
Intersubband Excitations at Finite Temperatures and their Roles in Different Quasi-One-Dimensional Systems
}

\author{
Marcos R. S. Tavares* and Guo-Qiang Hai ${ }^{\dagger}$ \\ * Instituto de Física Teórica. Universidade Estadual Paulista. São Paulo, SP, 01405-900, Brazil \\ ${ }^{\dagger}$ Instituto de Física de São Carlos, Universidade de São Paulo, São Carlos, SP, 13560-970, Brazil
}

Received on 4 April, 2005

\begin{abstract}
We theoretically study many-body excitations in three different quasi-one-dimensional (Q1D) electron systems: (i) those formed on the surface of liquid Helium; (ii) in two coupled semiconductor quantum wires; and (iii) Q1D electrons embedded in polar semiconductor-based quantum wires. Our results show intersubband coupling between higher subbands and the two lowest subbands affecting even the lower energy intersubband plasmons on the liquid Helium surface. Concerning the second system, we show a pronounced extra peak appearing in the intersubband impurity spectral function for temperatures as high as $20 \mathrm{~K}$. We finally show coupled intersubband plasmon-phonon modes surviving for temperatures up to $300 \mathrm{~K}$.
\end{abstract}

Keywords: Intersubband excitations; Many-body excitations; Quasi-one-dimensional (Q1D) electron systems

\section{INTRODUCTION}

Since the seminal work by Goñi et al., [1] where fundamental many-body excitations in GaAs/AlGaAs semiconductorbased quantum wires were experimentally probed, collective (plasmons) and single-particle (particle-hole) excitations in Q1D electron systems have been extensively studied. [2,3] Those experimental results were concerned to the inelastic light (Raman) scattering in Q1D electron systems and have been interpreted within the random-phase approximation (RPA) in the framework of the Fermi liquid (FL) theory. Also, Q1D electron gas confined on the surface of liquid helium has also been realized in laboratory by bending the liquid helium surface. [4] In some sense, this Q1D system is similar to those in semiconductor quantum wires, nanotubes and metallic chains where many-body effects can be studied.

Several papers have also been published concerning collective excitations in coupled double semiconductor quantum wires. In particular, a new kind of tunneling spectroscopy in these systems was able to check out the Luttinger liquid (LL) behaviour of the electrons in extremely clean limit at very low temperatures. [5] But, so far, it is still a puzzle in clarifying the conditions under which the 1D electron system embedded in semiconductor quantum wires behaves as a LL. We mention that effects of the intersubband (or interwire) excitations are believed to be enough to disable the LL behaviour, provided the system looses its strict one-dimensional character. [6]

This paper will be focussing on the intersubband manyparticle excitations occurring in different kinds of electronic Q1D systems that were mentioned above. Concerning to those formed on the surface of liquid Helium our results show that there is only one observable intrasubband plasmon mode, but many intersubband modes. All observable intersubband modes are related to the first and second subbands. Moreover, we show that strong Landau damping occurs when the plasmon energy approaches the energy difference between two subbands, leading to gaps in the plasmon spectra. [7]

Our results also indicate room temperature effects regarding coupled intersubband plasmon-phonon modes in a polar semiconductor based multisubband quantum wires. We show intersubband plasmon-phonon modes being observed for temperatures up to $300 \mathrm{~K}$. [8]

We finally study the impurity induced optical properties in Q1D electron systems embedded in two coupled semiconductor quantum wires at finite temperature. Our results indicate that the intersubband (or inter-wire tunneling) induced singleparticle excitations lead to a nonmonotonic impurity spectral function. [6]

This paper is organized as follows. In Sec. II we show our working formulae. Sec. III shows the numerical results. We summarize our results in Sec. IV.

\section{THEORETICAL FORMULATION}

\section{A. Spectral weight function}

A safer manner to analyze many-particle excitations at finite temperatures in multisubband systems is to look directly at the so-called spectral weight function [9]

$$
S(q, \omega)=-\sum_{\alpha \beta} \operatorname{Im}\left[\varepsilon_{b}(\omega) \chi_{\alpha \beta}(q, \omega)\right]
$$

where $\alpha \equiv\left(i, i^{\prime}\right)$ and $\beta \equiv\left(j, j^{\prime}\right)$ are the coupled indices with the subband indices $i, i^{\prime}, j$, and $j$ '. In Eq. (1), the frequency-dependent dielectric function $\varepsilon_{b}=1+$ $\left[\left(\omega_{T O}\right)^{2}-\left(\omega_{L O}\right)^{2}\right] /\left[\omega^{2}-\left(\omega_{L O}\right)^{2}+i \omega \eta\right]$ models the polarization of the background polar semiconductor, with $\omega_{L O}$ and $\omega_{T O}$ being the longitudinal-optical (LO) and the transverse-optical (TO) phonon frequencies, respectively.

The density-density correlation function $\chi_{\alpha \beta}(q, \omega)$ in Eq. (1) is obtained through the generalized random-phaseapproximation (RPA) equation

$$
\sum_{l l^{\prime}} \varepsilon_{l l^{\prime}, i i^{\prime}}(q, \omega) \chi_{l l^{\prime}, j j^{\prime}}(q, \omega)=\Pi_{i i^{\prime}}(q, \omega) \delta_{i j} \delta_{i^{\prime} j^{\prime}}
$$


which involves the electronic dielectric function $\varepsilon_{\alpha \beta}(q, \omega)$ and the 1D multisubband irreducible polarizability $\Pi_{\beta}(q, \omega)$. [10] For Q1D electrons confined on the surface of liquid Helium, Maxwell-Boltzmann statistics is used instead of Fermi-Dirac in calculating $\Pi_{\beta}(q, \omega)$. [7]

The electronic dielectric function in Eq. (2) is written as a summation of the two contributions: the electron-phonon interaction (first term) and the electron-electron interaction (second term). It has the following form

$$
\varepsilon_{\alpha \beta}(q, \omega)=\varepsilon_{b}(\omega) \delta_{\alpha \beta}-V_{\alpha \beta}(q) \bullet \Pi_{\beta}(q, \omega),
$$

where $V_{\alpha \beta}(q)$ is the Coulomb electron-electron (e-e) bare interaction in the 1D geometry. [10] Notice that, by setting $\varepsilon_{b}=1$ in Eq. (3), i.e. without electron-phonon interaction, the dielectric function of the Q1D electron gas within the RPA is recovered.

When the confinement potential in the $y$-direction is symmetric, e-e Coulomb interaction $V_{\alpha \beta}(q)=0$ when $i+i{ }^{\prime}+j+j$ ' is an odd number. As a consequence, the spectral weight function can be treated separately into two parts: [8]

$$
S(q, \omega)=S^{\text {even }}(q, \omega)+S^{\text {odd }}(q, \omega) .
$$

By carefully studying this function, one is able to map the $\omega-q$ plane and plot the dispersion relation of collective and single-particle excitations. By showing all excitation modes through the peaks in $S(q, \omega)$, one provides a very efficient guide of what should be observable in the experiments.

\section{B. Impurity spectral function}

Here, two parallel wires (with square-well type confinement potential) in the $x$-direction are considered with zero thickness in the $z$-direction. In the $y$-direction, the two wires are of $30 \mathrm{~nm}$ and $20 \mathrm{~nm}$ widths, respectively, separated by a 3-nm AlGaAs barrier of $300 \mathrm{meV}$ height. The energy gap between the first and the second subbands is $\omega_{12}=5.36 \mathrm{meV}$. We consider the impurity localized at the center of the barrier. Such a system is also similar to a type-II quantum wire heterostructures, where valence holes are localized between two parallel 1D electron gases.

We model this system by an Anderson impurity localized below the continuum of the two subbands in the coupled quantum wires filled with electrons. We devoted special attention to the interwire element of the impurity spectral function

$$
A_{12}(\omega)=-2 \operatorname{Im} \int d t \exp (i \omega t) g_{12}(t)
$$

where $g_{12}(t)$ is the finite temperature impurity Greens function involving electronic transitions between the wires.

The tunneling between the wires is responsible for the existence of $g_{12}(t)$. [6]

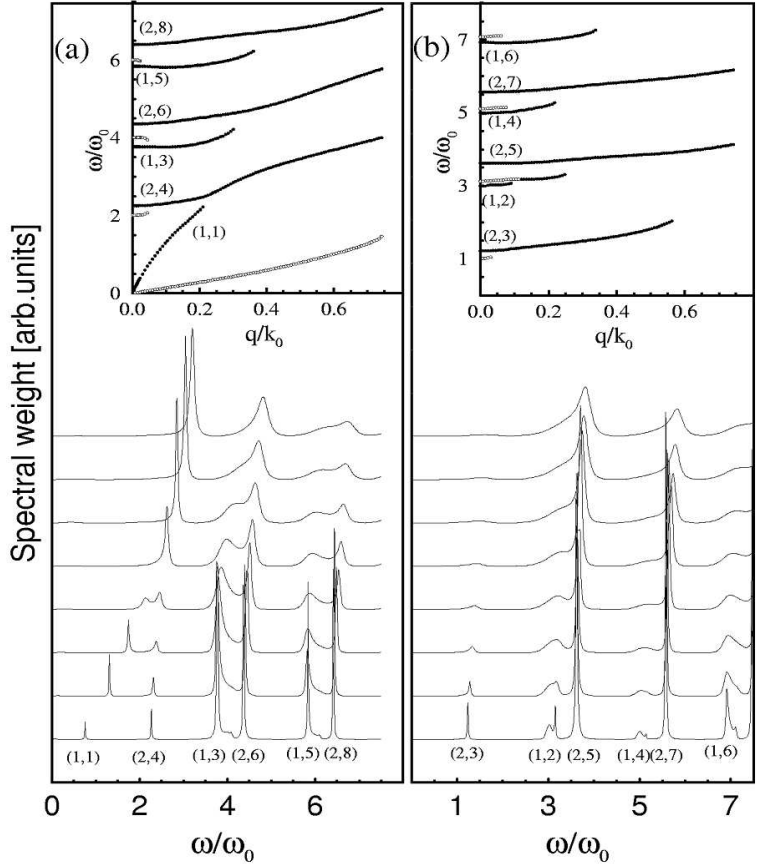

FIG. 1: The spectral weight (a) $S^{\text {even }}(q, \omega)$ and (b) $S^{\text {odd }}(q, \omega)$ of a Q1D channel formed over the liquid Helium at $T=0.4 \mathrm{~K}$ for different wave-vectors corresponding to those in the inset. The electron density is $N_{e}=10^{4} \mathrm{~cm}^{-1}$. The insets give the full dispersion relation of the plasmon modes $(n, m)$. The open-dots indicate the small peaks due to single-particle excitations.

\section{NUMERICAL RESULTS}

In Fig. 1, we plot the spectral weight function $S(q, \omega)$ for different $q$ values in a quantum wire channel formed on the surface of liquid Helium with an electron density $N_{e}=$ $10^{4} \mathrm{~cm}^{-1}$ and temperature $T=0.4 K$. The $q$ values correspond to those in the inset. Fig. 1(a) and 1(b) show the dispersion relations of the plasmon modes $(n, m)$ which have been obtained from the peaks in $S^{\text {even }}(q, \omega)$ and $S^{\text {odd }}(q, \omega)$, respectively. We also see small peaks in the full spectra due to single-particle excitations (SPEs) indicated by the open-dots in the insets. The lowest branch (open-dots) represents the intrasubband SPEs. With increasing $q$, the spectral weight of the intrasubband (intersubband) plasmon modes increases (decreases). We also observe the Landau damping induced gaps appearing around $\omega=n \omega_{0}$ at small $q$ where the SPEs are strong. Here, $\omega_{0}$ is the characteristic frequency describing the quantum wire confinement. We observe that the Landau damping becomes stronger when the plasmon mode $(n, m)$ approaches to the frequency $\omega=(n+1) \omega_{0}$, where the SPEs are stronger. [7]

We show in Fig. 2 the dispersion relations of the coupled 


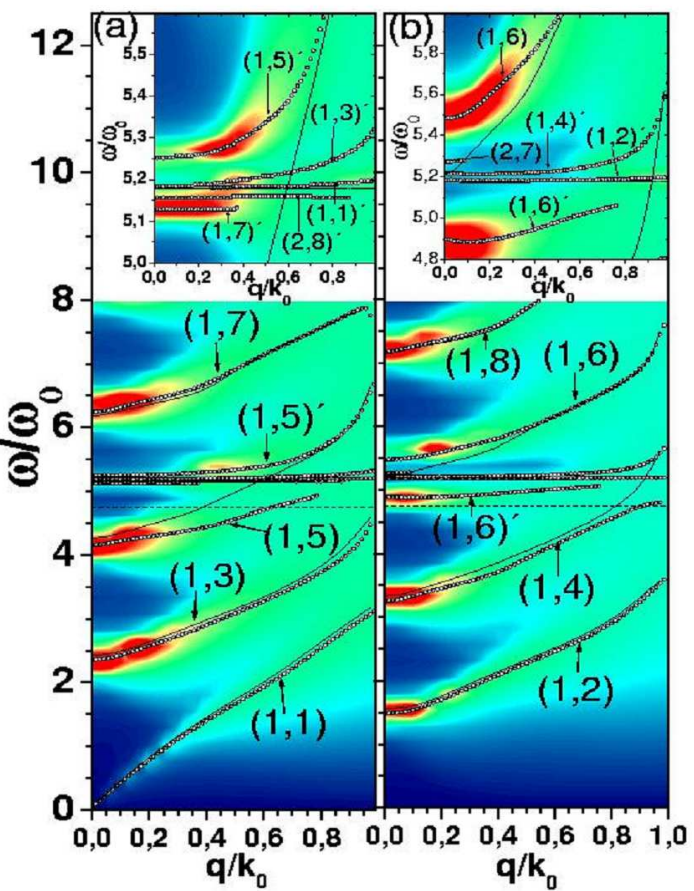

FIG. 2: The coupled plasmon-phonon dispersion relations in polar semiconductor based quantum wires at $T=50 \mathrm{~K}$ obtained from the peaks of (a) $S^{\text {even }}(q, \omega)$ and (b) $S^{\text {odd }}(q, \omega)$. The electron density is $N_{e}=10^{6} \mathrm{~cm}^{-1}$. Thin solid lines show the dispersion relations without electron-phonon interaction. The horizontal dashed lines indicate the frequencies of TO and LO phonons, $\omega_{T O}=4.75 \omega_{0}$ and $\omega_{L O}=5.17 \omega_{0}$, respectively. The inset shows the vicinity of $\omega_{L O}$.

plasmon-phonon modes (open-dot curves) in polar semiconductor based quantum wires. They are taken from the peaks in (a) $S^{\text {even }}(q, \omega)$ and (b) $S^{\text {odd }}(q, \omega)$ for different wave-vectors $q$. The temperature $T=50 \mathrm{~K}$ and the electron density $N_{e}=10^{6}$ $\mathrm{cm}^{-1}$. The colors indicate the intensity of the spectral weight calculated for all $\omega-q$ plane. They go from navy-blue (zero intensity) to red, which indicates a variation of 10 times in the spectral intensity. For $N_{e}=10^{6} \mathrm{~cm}^{-1}$, two subbands are occupied with the lowest having the most electron density. This helps us to identify the most weighted peaks indicated in Fig. 1. Extra coupled modes labelled as $(n, m)^{\prime}$ are better seen in the inset. They show up in the vicinity of $\omega_{L O}$ as a result of the electron-phonon interaction. Notice also that the branches above (below) $\omega_{L O}$ shift to higher (lower) frequencies when the electron-phonon interaction is considered. The presence of bulk LO-phonons in the system is responsible for such a shift. The upper panels in Fig.2 detail the vicinity of $\omega_{L O}$. The intensity scales in the upper panel are changed according to the change in the $\omega$-axis scale. In this region, only the modes related to electrons in the first subband are of noticeable intensities. But we show the less weighted extra mode $(2,8)^{\prime}$ appearing in Fig.2(a), while the mode $(2,7)$ is seen in Fig. 2(b). We mention that these modes are being seen at $T=50 \mathrm{~K}$ due to their strong phononlike character.

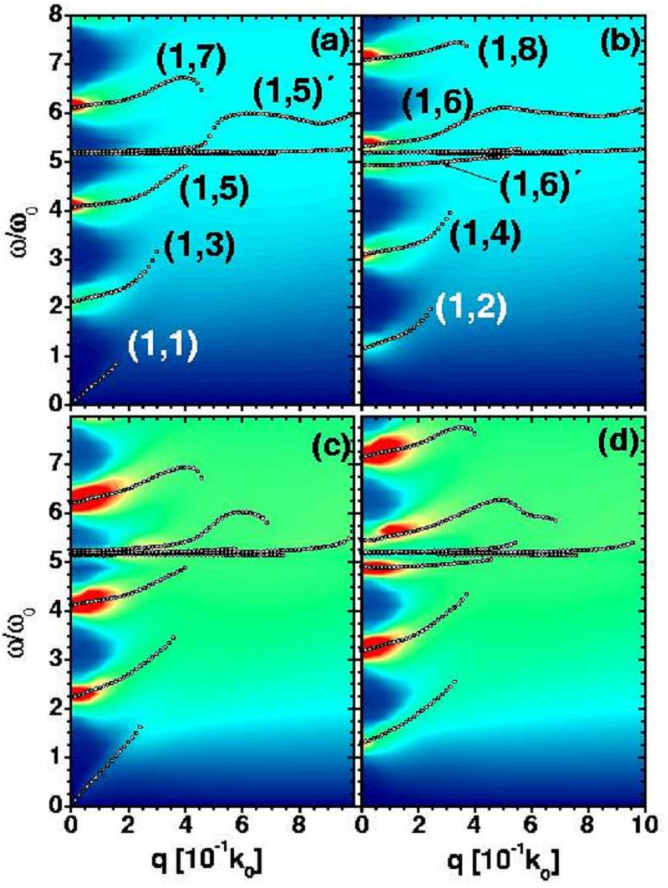

FIG. 3: Coupled intersubband plasmon-phonon modes $(1, n)$ obtained by mapping the spectral weight function (a and c) $S^{\text {even }}(q, \omega)$ and (b and d) $S^{\text {odd }}(q, \omega)$ at $T=300 \mathrm{~K}$. The electron density in (a) and (b) is $N_{e}=10^{6} \mathrm{~cm}^{-1}$, while $N_{e}=2.2 \times 10^{6} \mathrm{~cm}^{-1}$ in (c) and (d).

For the sake of completeness, we compare, in Fig. 3, the dispersion relations at $T=300 \mathrm{~K}$ for two different electron densities. We show in Figs. 3(a) and 3(b) the intersubband coupled modes $(1, n)$ for $N_{e}=10^{6} \mathrm{~cm}^{-1}$, while Figs. 3(c) and 3(d) show the same for $N_{e}=2.2 \times 10^{6} \mathrm{~cm}^{-1}$. Spectral weight intensities decrease as the wave-vector $q$ increases, but they are still clearly noticeable at $T=300 \mathrm{~K}$ (red regions) for both densities.

In Fig. 4(a), we show $A_{12}(\omega)$ in coupled asymmetric quantum wires at $T=2 \mathrm{~K}$ for different charge densities $N_{e}$. The figure shows the effects due to interwire SPEs. These effects lead to a non-monotonic impurity spectral function. $\mathrm{As}_{e}$ decreases, the narrower wire becomes less populated and the shoulders in the figures get affected. In Fig. 4(b), we show the temperature evolution of $A_{12}(\omega)$ for $N_{e}=10^{6} \mathrm{~cm}^{-1}$. Although the temperature suppresses the interwire excitations, their effects are observable up to $T=30 \mathrm{~K}$.

\section{CONCLUSIONS}

In summary, we investigated the plasmon modes of the Q1D electron systems on the surface of the liquid helium. We used a multisubband approach and treated the system as a classical nondegenerate gas obeying Maxwell-Boltzmann sta- 


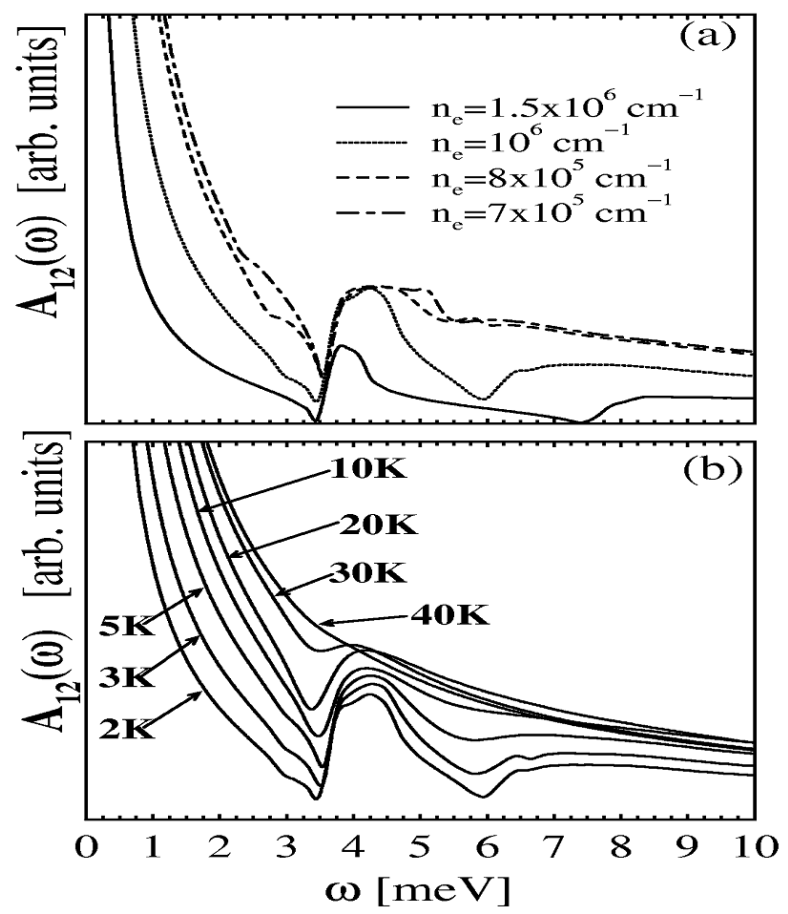

FIG. 4: (a) Interwire spectral function $A_{12}(\omega)$ at $T=2 \mathrm{~K}$ for different $N_{e}$ in coupled asymmetric quantum wires. As the density decreases, the narrower wire becomes less populated and the shoulders in the figure get affected. (b) Here we show the temperature evolution of $A_{12}(\omega)$ for $N_{e}=10^{6} \mathrm{~cm}^{-1}$. Interwire SPEs are observable in our calculation up to $T=30 \mathrm{~K}$.

tistics. We found strong intersubband plasmon modes related to the first two subbands. The SPEs in the system are responsible for strong Landau damping at frequencies $\omega=n \omega_{0}$ where gaps appear in the plasmon spectra. We also have mapped out the $\omega-q$ plane concerning coupled intersubband plasmonphonon modes at room temperatures in a multisubband Q1D quantum wires based on polar semiconductors. We remarkably found coupled intersubband plasmon-phonon modes related to the first subband surviving up to temperatures as high as $300 \mathrm{~K}$. We finally studied the non-diagonal (interwire) element of the spectral function matrix of a localized impurity in a coupled double quantum wires. We show that the tunneling between the two asymmetric wires induces interwire (or intersubband) particle-hole excitation effects leading to a pronounced peak in the impurity spectral function up to $20 \mathrm{~K}$.

This work was supported by FAPESP and CNPq.
[1] A. Goñi, A. Pinczuk, J.S. Weiner, J.M. Calleja, B.S. Dennis, L.N. Pfeiffer, and K.W. West, Phys. Rev. Lett. 67, 3298 (1991).

[2] B.Y.-K. Hu and S. Das Sarma Phys. Rev. B 48, 5469 (1993).

[3] J. Voit, Rep. Prog. Phys. 58, 977 (1995).

[4] Yu. Z. Kovdrya and V.A. Nikolaenko, Fizika Nizkikh Temperature 18, 1278 (1992).

[5] E.H. Hwang and S. Das Sarma, Phys. Rev. B 58 R1738 (1998); M.R.S. Tavares and G.-Q. Hai , Phys. Rev. B 61, 7564 (2000); and O.M. Auslaender et al., Science 295, 825 (2002).
[6] M.R.S. Tavares, G.-Q Hai, and G.E. Marques, Physica E 21, 479 (2004).

[7] M.R.S. Tavares, G.-Q. Hai, F.M. Peeters, and N. Studart, Phys. Rev. B 68, 140504(R) (2003).

[8] M.R.S. Tavares, Phys. Rev. B 71, to appear (2005).

[9] For a rewiew, see G. D. Mahan, Many particle Physics (Plenum, New York, 1981), second edition.

[10] M.R.S. Tavares and G.-Q. Hai , Phys. Rev. B 61, 7564 (2000). 